\title{
Neuromyelitis optica and pregnancy during therapeutic $B$ cell depletion: infant exposure to anti-AQP4 antibody and prevention of rebound relapses with low-dose rituximab postpartum
}

Multiple Sclerosis Journal

19(II) 1544-1547

(C) The Author(s) 2013

Reprints and permissions:

sagepub.co.uk/journalsPermissions.nav DOI: 10.1 I $77 / 1352458513498125$ msj.sagepub.com

@SAGE

\author{
M Ringelstein', J Harmel', F Distelmaier', J Ingwersen', T Menge', \\ K Hellwig', B Kieseier', E Mayatepek², H-P Hartung', \\ T Kuempfel ${ }^{4}$ and O Aktas ${ }^{1}$
}

\begin{abstract}
Neuromyelitis optica (NMO) predominantly affects women, some in childbearing age, and requires early therapeutic intervention to prevent disabling relapses. We report an anti-AQP4 antibody-seropositive patient who became pregnant seven months after low-dose (I00 mg) rituximab application. Pregnancy showed no complications, and low-dose rituximab restarted two days after delivery resulted in neurological stability for 24 months. Remarkably, her otherwise healthy newborn presented with anti-AQP4 antibody and reduced B lymphocyte counts in umbilical cord blood, which normalized three months later. Confirming and extending previous reports, our case suggests that low-dose rituximab might be compatible with pregnancy and prevent rebound NMO disease activity postpartum.
\end{abstract}

\section{Keywords}

Neuromyelitis optica, pregnancy, rituximab, relapse prevention, antibody transfer

Date received: 20 February 20I3; revised: I2 June 20I3; accepted: 26 June 2013

\section{Introduction}

Neuromyelitis optica (NMO) shows a marked female preponderance and may affect patients in childbearing age, often suffering from disabling relapses by inflammation of the optic nerve and the spinal cord. ${ }^{1}$ According to retrospective case studies, patients with $\mathrm{NMO}$ are prone to relapses shortly after delivery, ${ }^{2,3}$ similar to multiple sclerosis (MS). ${ }^{4}$ Rituximab is a chimeric monoclonal anti-CD20 antibody shown to prevent NMO relapses in independent retrospective case series and prospective long-term studies. ${ }^{5,6}$ Recently, the clinical course of NMO in a pregnant woman who received rituximab shortly before conception and experienced two relapses 10 days and two months after delivery has been described. ${ }^{7}$ Here, we report the case of an NMO patient who became pregnant following low-dose rituximab and remained stable by early postpartum rituximab treatment.

\section{Case report}

The previously healthy 18 -year-old woman had developed a subacute Brown-Séquard syndrome in October 2008 after human papillomavirus- and Neisseria meningitidis vaccinations as previously reported (case no. 1 in the report by Menge et $\left.a .^{8}\right)$. Three months later, she suffered from severe left-sided optic neuritis $(\mathrm{ON})$, spastic paraparesis and bladder dysfunction, and NMO was diagnosed (antiaquaporin 4 (AQP4) antibody ratio: 48.5 , measured by

\footnotetext{
'Department of Neurology, Medical Faculty, Heinrich-Heine-University Düsseldorf, Germany.

2Department of General Pediatrics, Neonatology and Pediatric

Cardiology, University Children's Hospital, Medical Faculty, HeinrichHeine-University Düsseldorf, Germany.

${ }^{3}$ Department of Neurology, St. Josef-Hospital, Ruhr University Bochum, Germany.

${ }^{4}$ Institute of Clinical Neuroimmunology, Ludwig-Maximilians-University

Munich, Germany.

Corresponding author:

Orhan Aktas, Department of Neurology, Medical Faculty, Heinrich-

Heine-University Düsseldorf, Moorenstraße 5, 40225 Düsseldorf,

Germany.

Email: orhan.aktas@uni-duesseldorf.de
} 


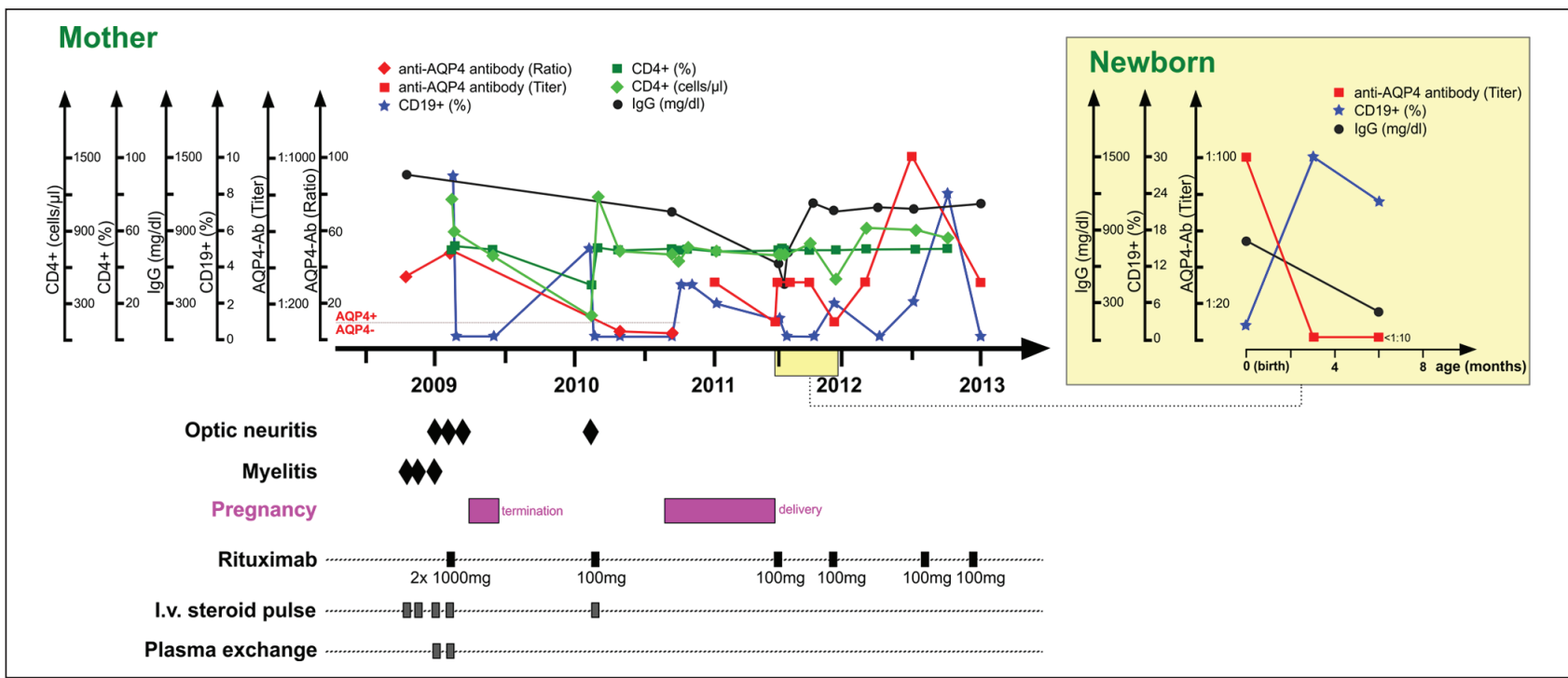

Figure. Disease course, laboratory parameters and therapy of NMO patient. Shown are relapses (diamonds), acute and long-term treatments (intravenously applied high-dose steroid therapy, $1000 \mathrm{mg}$ per day for three to five days; plasma exchange, rituximab), and the two pregnancies of the NMO patient over a follow-up period of 52 months. No relapses occurred during pregnancy and postpartum on a low-dose rituximab treatment seven months before pregnancy and two days after delivery. Moreover, anti-AQP4 antibody ratios or titers, CDI9+ B cell counts, CD4+ T cell counts and percentages and IgG serum values are presented. Two different anti-AQP4 antibody detection assays were applied in the mother (radioimmunoprecipitation assay until August 20 I0; cellbased immunofluorescence assay thereafter). (Inset) Laboratory parameters of newborn. Reduced B cell counts and increased antiAQP4-antibodies in the umbilical cord blood of the child normalized within three months. Anti-AQP4 antibodies were determined by cell-based immunofluorescence assay (see above).

NMO: neuromyelitis optica; AQP4: aquaporin 4; IgG: immunoglobulin G.

radioimmunoprecipitation; Figure). After relapse therapy with high-dose steroids and plasma exchange, rituximab was initiated (1000 mg twice within two weeks) and the patient partially improved. Shortly after, she became inadvertently pregnant for the first time and medically indicated termination was performed at 19 years of age (in June 2009). Eight months later, she developed another severe left-sided ON, leading to blindness despite therapy with high-dose steroids. At this time (February 2010), CD19+ B cell count was at $5 \%$ (normal range, 5-24). In light of growing experiences with adaptation of rituximab therapy regimen, ${ }^{9}$ our patient was treated with low-dose rituximab (single application of $100 \mathrm{mg}$ ) leading to persisting B cell depletion and anti-AQP4 antibody disappearance until the age of 20 years (August 2010). One month later, seven months after the last rituximab infusion, the patient became pregnant again (CD19+ B cell count: 3\%). During pregnancy, no relapses occurred, but anti-AQP4 antibody reappeared (now measured by cell-based immunofluorescence (CBA)), while CD19+ B cells further decreased (10/2010: 3\%; 01/2011: 2\%; 06/2011: 1\%, Figure). After 38 weeks of pregnancy, a healthy, normally developed male child was delivered by caesarean section (length $50 \mathrm{~cm}$, weight $3220 \mathrm{~g}$ ). Remarkably, analysis of the newborn's umbilical cord blood (Figure, inset) showed decreased CD19+ B cells ( $2 \%$, normal range, 5-24) and increased anti-AQP4 antibody titers (1:100; $\mathrm{CBA})$; however, the baby did not show any specific neuro- logical deficits. The mother presented reduced B cell counts $(1 \%)$ and reduced immunoglobulin $\mathrm{G}$ ( $\mathrm{IgG}$ ) serum levels (608 mg/dl; normal range, 700-1600), but still increased anti-AQP4 antibody titers (1:100, CBA), comparable to her offspring. In light of these findings and the patient's disabling disease course prior to pregnancy, she was treated again with rituximab $(100 \mathrm{mg})$ two days after delivery. Nine days thereafter, the patient presented with fever, lower abdominal pain and fatigue. Endometritis or cystitis was suspected and successfully treated with metronidazol, sultamicillin and oxytocin. Since delivery, no NMO relapses occurred for the following 24 months on low-dose rituximab $(100 \mathrm{mg})$ treatments every five to seven months, while antiAQP4 antibody titers persisted (Figure). Anti-AQP4 antibody testing of the baby turned negative and CD19+ B cells normalized at three and six months after birth. Except for a mild rhinitis and conjunctivitis at the age of one month, the child developed normally, and no neurological or other abnormalities occurred until the current age of 24 months.

\section{Comment}

Our case confirms and extends recent observations on $\mathrm{NMO}$, pregnancy and rituximab: Recent studies indicate that NMO patients are prone to relapses during the first and second trimester after delivery, ${ }^{2,3}$ similar to patients with relapsing MS. ${ }^{4}$ The underlying mechanisms, however, are 
unclear. It has been suggested that withdrawal of pregnancy-related immunological tolerance mechanisms, mediated by regulatory $\mathrm{T}$ cells, ${ }^{10}$ induce an immunological and clinical rebound effect. Considering the severity of NMO relapses, preventive therapy in the postpartum phase may therefore prove justified and our case suggests a rationale for such an intervention early after delivery. Our patient had received rituximab seven months before pregnancy and two days after delivery at a dose of $100 \mathrm{mg}$ and remained stable throughout these periods. Remarkably, the newborn had reduced B lymphocyte levels in the umbilical blood, returning to normal after three months, indicating a sustained but transient biological effect of even low-dose rituximab on the infant's immune system (Figure). Except for a suspected abdominal infection requiring antibiotics nine days after caesarean delivery, no safety signals were detected for the mother. These findings support previous experiences with an NMO patient with inadvertent conception one week after application of high-dose rituximab (1000 mg twice within two weeks). ${ }^{7}$ In that report, the child developed normally during follow-up for 15 months after birth. The mother was clinically stable during pregnancy, but had not been treated upon delivery and experienced two severe relapses 10 days and two months thereafter. ${ }^{7}$

In our patient, the correlation between anti-AQP4 antibody titers and clinical disease activity, as previously observed, ${ }^{11}$ as well as the relation between rituximab administration and anti-AQP4 antibody, remained ambiguous. Rituximab may disrupt T-B lymphocyte interactions, which is beneficial in MS and NMO, while we were not able to establish a solid correlation between anti-AQP4 antibody titers, clinical relapses and drug application. Thus, our data corroborate recent suggestions for studies on biomarker validation in NMO. ${ }^{12}$

Finally, we here show that anti-AQP4 antibody can be transferred from the mother to the child but may not have harmful effects in the latter. The full-term male child showed no neurological or other abnormalities during 24 months of follow-up, in line with earlier reports on offspring of NMO patients. ${ }^{2,3}$ As the baby was seronegative for anti-AQP4 antibody three months after delivery, we assume a passive transfer from the seropositive mother to the child without any obvious impact, similar to the passive antibody transfer to newborns in myasthenia gravis. ${ }^{13}$

In conclusion, our case suggests that anti-AQP4 antibody may pass the blood-placenta barrier without causing neurological deficits in the newborn child, and that early maternal postpartum rituximab therapy may be an option to prevent rebound NMO disease activity after delivery.

\section{Conflict of interest statement}

- Marius Ringelstein has received speaker honoraria from Novartis and travel reimbursement from Bayer Schering and Biogen Idec.

- Jens Harmel has received speaker honoraria from Novartis.
- Felix Distelmaier and Jens Ingwersen have nothing to declare.

- Til Menge has received honoraria and travel support from Bayer Schering Pharma, Biogen Idec, Teva and Merck Serono.

- Kerstin Hellwig has received honoraria for lecturing and travel expenses for attending meetings and received financial research support from Bayer Schering, Biogen Idec, Merck Serono, Novartis, Sanofi-Aventis and Teva. Currently she is supported by the German Research Council (DFG, grant HE 6841/1-1).

- Bernd Kieseier has received honoraria for lecturing and travel expenses for attending meetings and received financial research support from Bayer Schering, Biogen Idec, Merck Serono, Novartis, Sanofi Aventis and Teva. He has served or serves as consultant for Biogen Idec, Medac, Sanofi Aventis and Teva. He received grants from the German Ministry for Education and Research and the "German Competence Network Multiple Sclerosis."

- Ertan Mayatepek has received grants from the German Research Foundation (DFG 575, KFO 217) and honoraria for lectures by Swedish Orphan Biovitrum and Nestlé.

- Hans-Peter Hartung has received grants from Walter-and-IlseRose-Stiftung, the Eugène Devic European Network (EDEN, EU-FP7) and the German Ministry for Education and Research, honoraria for consultancy by Bayer Health Care, Biogen Idec, Genzyme, Novartis, Teva, Sanofi Aventis and Roche and holds patents.

- Tania Kuempfel reports personal compensations (speaker honoraria) and travel support from Bayer Schering Pharma, Teva, Sanofi Aventis, Novartis Pharma, Merck Serono and Biogen Idec. TK has received grant support by Novartis Pharma.

- Orhan Aktas has received grants from the German Research Foundation(DFG SFB974, GRK1033), Eugène Devic European Network (EDEN, EU-FP7), German Ministry for Education and Research, Schaufler Foundation, honoraria for lectures by Novartis, Bayer Schering, Teva, Biogen Idec and Merck Serono, holds patents and has received travel/accommodation/ meeting expenses from Novartis, Bayer Schering and Merck Serono.

\section{Funding}

This research received no specific grant from any funding agency in the public, commercial, or not-for-profit sectors.

\section{References}

1. Wingerchuk DM, Lennon VA, Lucchinetti CF, et al. The spectrum of neuromyelitis optica. Lancet Neurol 2007; 6: 805-815.

2. Bourre B, Marignier R, Zephir H, et al. Neuromyelitis optica and pregnancy. Neurology 2012; 78: 875-879.

3. Kim W, Kim SH, Nakashima I, et al. Influence of pregnancy on neuromyelitis optica spectrum disorder. Neurology 2012; 78: 1264-1267.

4. Confavreux C, Hutchinson M, Hours MM, et al. Rate of pregnancy-related relapse in multiple sclerosis. Pregnancy in Multiple Sclerosis Group. N Engl J Med 1998; 339: 285-291.

5. Jacob A, Weinshenker BG, Violich I, et al. Treatment of neuromyelitis optica with rituximab: Retrospective analysis of 25 patients. Arch Neurol 2008; 65: 1443-1448. 
6. Kim SH, Kim W, Li XF, et al. Repeated treatment with rituximab based on the assessment of peripheral circulating memory B cells in patients with relapsing neuromyelitis optica over 2 years. Arch Neurol 2011; 68: 1412-1420.

7. Pellkofer HL, Suessmair C, Schulze A, et al. Course of neuromyelitis optica during inadvertent pregnancy in a patient treated with rituximab. Mult Scler 2009; 15: 1006-1008.

8. Menge T, Cree B, Saleh A, et al. Neuromyelitis optica following human papillomavirus vaccination. Neurology 2012; 79: 285-287.

9. Greenberg BM, Graves D, Remington G, et al. Rituximab dosing and monitoring strategies in neuromyelitis optica patients:
Creating strategies for therapeutic success. Mult Scler 2012; 18: 1022-1026.

10. Trowsdale J and Betz AG. Mother's little helpers: Mechanisms of maternal-fetal tolerance. Nat Immunol 2006; 7: 241-246.

11. Jarius S, Aboul-Enein F, Waters P, et al. Antibody to aquaporin-4 in the long-term course of neuromyelitis optica. Brain 2008; 131: 3072-3080.

12. Chanson JB, de Seze J, Eliaou JF, et al. Immunological follow-up of patients with neuromyelitis optica: Is there a good biomarker? Lupus 2013; 22: 229-232.

13. Lefvert AK and Osterman PO. Newborn infants to myasthenic mothers: A clinical study and an investigation of acetylcholine receptor antibodies in 17 children. Neurology 1983; 33: 133-138. 